\title{
Annullatoren von Pfisterformen über semilokalen Ringen
}

\author{
Ricardo Baeza und Manfred Knebusch
}

\section{§1. Einleitung}

Mit der vorliegenden Untersuchung setzen wir unser in den Arbeiten [6], $[1, \S 1]$ und [2] begonnenes Studium der Pfisterformen über semilokalen Ringen fort. Wir benutzen hier dieselben Bezeichnungen wie in den drei genannten Arbeiten bis auf den unwesentlichen Unterschied, daß alle symmetrisch bilinearen und quadratischen Räume in dieser Arbeit nicht nur projektive, sondern freie Moduln sein sollen.

Ohne Zweifel hat auch über semilokalen Ringen eine möglichst genaue Kenntnis der Eigenschaften von Pfisterformen zentrale Bedeutung für den weiteren Aufbau einer Theorie der quadratischen und (symmetrisch) bilinearen Formen, wie dies über Körpern eindrucksvoll durch Arbeiten von Pfister, Arason, Elman und Lam belegt wird.

$A$ ist in dieser Arbeit stets ein kommutativer Ring mit Eins, der semilokal ist, d.h. nur endlich viele maximale Ideale hat. $W(A)$ bezeichnet den Wittring der (freien) bilinearen Räume über $A$ und $W q(A)$ die Wittgruppe der (freien) quadratischen Räume über $A$.

Wie in [1] bezeichnen wir für Elemente $a, b$ aus $A$ mit 1-4ab Einheit den binären quadratischen Raum $\left[\begin{array}{ll}a & 1 \\ 1 & b\end{array}\right]$ kurz mit $[a, b]$. In $[6, \S 4]$ wurde unter einer kleinen Einschränkung für $A$ gezeigt, daß das Annullatorideal eines quadratischen nicht hyperbolischen Pfisterraumes

$$
F=\left(1, a_{1}\right) \otimes \cdots \otimes\left(1, a_{n}\right) \otimes[1, b],
$$

$n \geqq 0$, in dem Wittring $W(A)$ durch eigentliche binäre Räume erzeugt wird, also durch Räume $(1,-c)$ mit $c$ in der Gruppe $D^{*}(F)$ der durch $F$ dargestellten Einheiten. Für Körper einer Charakteristik $\neq 2$ wurde dieser Satz schon früher von E. Witt bemerkt [unveröffentlicht].

Sei nun

$$
\varphi=\left(1, a_{1}\right) \otimes \cdots \otimes\left(1, a_{n}\right) \quad(n \geqq 1)
$$

ein bilinearer Pfisterraum über $A$, dessen Bild in $W(A)$ nicht Null ist. Das Ziel der vorliegenden Arbeit ist die Bestimmung des Annullators $\mathfrak{A}(\varphi)$ von $\varphi$ in dem $W(A)$-Modul $W q(A)$. Ersichtlich ist $\mathfrak{Q}(\varphi)$ ein Untermodul von $W q(A)$. Wir bezeichnen mit $\varphi^{\prime}$ den reinen Anteil des Pfisterraumes $\varphi$, also den nach [2] durch die Zerlegung

$$
\varphi \cong(1) \perp \varphi^{\prime}
$$


bis auf Isomorphie eindeutig bestimmten Raum. Unser Hauptresultat besagt, daß $\mathfrak{A}(\varphi)$ als $W(A)$-Modul durch binäre Räume erzeugt wird sofern $A$ keinen Restklassenkörper mit nur 2 Elementen besitzt (Satz 6.1). Weiter zeigen wir, daß ein binärer quadratischer Raum $E$ mit $\varphi \otimes E \sim 0$ sich in der Gestalt

$$
E \cong(c) \otimes\left[1,-\gamma^{2}-\gamma-n(v)\right]
$$

schreiben läßt mit $c$ in $A^{*}, \gamma$ in $A$ und $v$ in $\varphi^{\prime}\left\{A^{*}=\right.$ Gruppe der Einheiten von $A$; $n(v)=$ Wert der Bilinearform auf $(v, v)\}$ und unter milden Einschränkungen für $A$ sogar in der Gestalt

$$
E \cong(c) \otimes[1,-n(v)]
$$

mit $v$ in $\varphi^{\prime}$ und $n(v)$ Einheit (Satz 5.1, Satz 5.2).

Zum Beweis benötigen wir als technisches Hilfsmittel einige „Transversalitätssätze“, die wir in $\$ 2$ und $\S 4$ zusammenstellen. Diese Transversalitätssätze dürften auch bei der Behandlung anderer Probleme über quadratische Formen von Nutzen sein. Zum Beispiel hat Hermann Bauer, ein Doktorand in Regensburg, kürzlich mit ihrer Hilfe festgestellt, daß über einem semilokalen Ring, in dem 2 Einheit ist, einige wichtige von Elman und Lam in [4] für Pfisterformen über Körpern bewiesene Aussagen richtig-bleiben, nämlich der Satz 3.2 dort über die „binäre Verbindlichkeit“ der Produktdarstellungen einer Pfisterform, und die Teilbarkeitssätze 1.4 und 2.7. Auch sei vermerkt, daß der in $[1, \S 1]$ bewiesene Satz, daß eine isotrope quadratische Pfisterform hyperbolisch ist, sich mit dem Transversalitätssatz 2.7 in $\$ 2$ wesentlich schneller herleiten läßt als in [1].

Im Hinblick auf weitere zu erwartende Anwendungen von Transversalitätssätzen erschien es uns angebracht, in $\$ 2$ und $\$ 4$ an einigen Stellen etwas mehr zu beweisen, als wir in der vorliegenden Arbeit brauchen.

Es bleibt zu fragen, ob das Annullatorideal eines bilinearen Pfisterraumes $\varphi$ in $W(A)$ durch eigentliche binäre Räume $(1,-a)$ erzeugt wird, und weiter, für welche Einheiten $a$ von $A$ der $\operatorname{Raum}(1,-a)$ durch $\varphi$ annulliert wird. Diese beiden Fragen sind unseres Wissens noch völlig offen, abgesehen von dem hier nicht mehr interessierenden Falle, daß 2 Einheit in $A$ ist, der schon in der Arbeit [6] erledigt wurde. Zur Verdeutlichung der Schwierigkeit, die einer Beantwortung selbst der zweiten Frage entgegensteht, bringen wir ein Beispiel.

Sei $\varphi=8 \times(1)$. Weiter sei $h$ eine natürliche Zahl, für die $2 h-1$ und $4 h-1$ in $A$ Einheiten sind. (Solche Zahlen $h$ gibt es zu jedem semilokalen Ring $A$.) Schließlich sei $a$ eine Einheit von $A$, die sich in der Form

$$
a=x^{2}+x y+y^{2} h
$$

mit $x, y \in A$ schreiben läßt und nicht in dem Teilring $A^{(2)}$ liegt, der von den Quadraten in $A$ erzeugt wird. Der quadratische Raum $(1,-a) \otimes[1, h]$ ist hyperbolisch. Daraus folgt mit dem im Beweis von Lemma 3.2 in [7] benutzten Verfahren, daß der bilineare Raum $\varphi \otimes(1,-a)=8 \times(1,-a)$ in $W(A)$ das Bild Null hat. Jedoch wird die Einheit $a$ nicht von dem $\operatorname{Raum} \varphi$ dargestellt.

\section{§ 2. Transversalitätssätze}

Wir machen zunächst eine Bemerkung über Basen von quadratischen Räumen über unserem semilokalen Ring $A$. Die quadratische Form eines quadratischen 
Raumes bezeichnen wir gewöhnlich mit $q$ und die zugehörige symmetrische Bilinearform mit $B$. Es ist also $B(x, y)=q(x+y)-q(x)-q(y)$.

Definition 2.1. Sei $E$ ein quadratischer Raum $\neq 0$ über $A$ und $n$ seine Dimension. Eine kanonische Basis von $E$ ist bei geradem $n=2 m$ eine Basis

des Moduls $E$ über $A$ mit

$$
\left\{e_{1}, f_{1}, \ldots, e_{m}, f_{m}\right\}
$$

$$
B\left(e_{i}, e_{j}\right)=B\left(f_{i}, f_{j}\right)=B\left(e_{i}, f_{j}\right)=0
$$

für $i \neq j$, und $B\left(e_{i}, f_{i}\right)=1$ für jedes $i$. Die Elemente $1-4 q\left(e_{i}\right) q\left(f_{i}\right)$ müssen dann Einheiten von $A$ sein. Ist $n=2 m+1$ ungerade und somit insbesondere 2 Einheit in $A$, so verstehen wir unter einer kanonischen Basis von $E$ eine Basis

$$
\left\{e_{1}, f_{1}, \ldots, e_{m}, f_{m}, g\right\}
$$

bei der die $e_{i}, f_{j}$ den obigen Beziehungen genügen, und $g$ auf allen anderen Basisvektoren senkrecht steht. $g$ muß dann strikt anisotrop sein, d.h. $q(g)$ ist Einheit. Eine kanonische Basis $\left\{e_{1}, f_{1}, \ldots, e_{m}, f_{m}\right\}$ bzw. $\left\{e_{1}, f_{1}, \ldots, e_{m}, f_{m}, g\right\}$ heiße strikt kanonisch, wenn alle Vektoren $e_{i}, f_{j}$ strikt anisotrop sind.

Es ist wohlbekannt und leicht zu zeigen, daß jeder quadratische Raum $E \neq 0$ eine kanonische Basis besitzt, bei der überdies die Vektoren $e_{i}$ alle strikt anisotrop sind. Wenn $\boldsymbol{A}$ nicht zu „klein“ ist, existiert sogar eine strikt kanonische Basis.

Lemma 2.2. Jeder Restklassenkörper $A / \mathrm{m}$ von $A$ enthalte mindestens 4 Elemente. $\{m$ durchläuft die endlich vielen maximalen Ideale von $A$.\} Dann hat jeder quadratische Raum $E \neq 0$ über $A$ eine strikt kanonische Basis.

Beweis. Weil $E$ sich orthogonal in Teilräume einer Dimension $\leqq 2$ zerlegen läßt, genügt es den Fall $\operatorname{dim} E=2$ zu betrachten. Indem wir die quadratische Form von $E$ mit einer geeigneten Einheit von $A$ multiplizieren, können wir erreichen, daß $E$ eine Basis $\{e, f\}$ mit $q(e)=B(e, f)=1$ hat. Sei $q(f)=b$. Wir suchen nun ein $\lambda$ in $A$, so daß die Werte

$$
q(\lambda e+f)=\lambda^{2}+\lambda+b, \quad B(e, \lambda e+f)=1+2 \lambda
$$

beide Einheiten sind. Das ist aufgrund des chinesischen Restsatzes möglich, weil alle Körper $A / \mathrm{m}$ mehr als 3 Elemente enthalten. Die Basis

$$
\left\{e,(1+2 \lambda)^{-1}(\lambda e+f)\right\}
$$

von $E$ ist strikt kanonisch. q.e.d.

Definition 2.3. Sei $\mathfrak{B}=\left\{z_{1}, \ldots, z_{n}\right\}$ eine Basis unseres quadratischen Raumes $E$ und sei

$$
x=\alpha_{1} z_{1}+\cdots+\alpha_{n} z_{n}
$$

ein Vektor aus $E$. Der Vektor $x$ heiße primitiv, falls der Modul $A x$ sich als direkter Summand von dem $A$-Modul $E$ abspalten läßt, also falls

$$
\alpha_{1} A+\cdots+\alpha_{n} A=A
$$

ist. $x$ heiße transversal zu der Basis $\mathfrak{B}$, falls sogar alle $\alpha_{i}$ Einheiten von $A$ sind. 
Wir kommen nun zu unserem ersten Transversalitätssatz.

Satz 2.4. Sei $x$ ein primitiver Vektor des quadratischen Raumes $E$ und $\mathfrak{B}$ eine strikt kanonische Basis von E. Der Ring A habe keine Restklassenkörper mit nur 2 Elementen und im Falle $\operatorname{dim} E=3$ auch keine Restklassenkörper mit nur 3.Elementen. Dann gibt es einen eigentlichen Automorphismus $\sigma$ von $E\left\{d\right.$.h. $\left.\sigma \in O^{+}(E)\right\}$, der $x$ in einen $z u \mathfrak{B}$ transversalen Vektor überführt.

Beweis. $\mathfrak{r}$ bezeichne das Jacobson-Radikal von $A$ und $\bar{E}$ bezeichne den Raum $E / \mathfrak{r} E$ über $A / \mathfrak{r}$. Das Bild $\overline{\mathfrak{B}}$ von $\mathfrak{B}$ in $\bar{E}$ ist eine kanonische Basis von $\bar{E}$ und das Bild $\bar{x}$ von $x$ in $\bar{E}$ ist ein primitiver Vektor von $\bar{E}$. Nun ist die natürliche Abbildung von $O^{+}(E)$ nach $O^{+}(\bar{E})$ surjektiv [8]. Haben wir also ein $\bar{\sigma}$ in $O^{+}(\bar{E})$ gefunden, so $\mathrm{da} \beta \bar{\sigma}(\bar{x})$ transversal zu $\overline{\mathfrak{B}}$ ist, so brauchen wir nur ein Urbild $\sigma$ von $\bar{\sigma}$ in $O^{+}(E)$ zu wählen. $\sigma(x)$ ist dann transversal zu $\mathfrak{B}$. Wir dürfen uns also auf den Fall $\mathfrak{r}=0$ zurückziehen und dann wegen

$$
\bar{E}=\prod_{\mathbf{m}} E / \mathrm{m} E, \quad O^{+}(\bar{E})=\prod_{\mathbf{m}} O^{+}(E / \mathrm{m} E)
$$

sogar auf den Fall, daß $A$ ein Körper ist. \{m durchläuft wieder die endlich vielen maximalen Ideale von $A$.

Sei also $A$ jetzt ein Körper. Es genügt, die Behauptung für $\operatorname{dim}(E) \leqq 4$ und im Falle $A=\mathbb{F}_{3}$ noch für $\operatorname{dim}(E)=5 \mathrm{zu}$ beweisen. Dann folgt die Behauptung für beliebiges $E$ durch Induktion nach der Dimension. Im Falle $\operatorname{dim}(E) \geqq 3$ genuigt es außerdem, ein $\sigma$ in $O(E)$ zu finden, das $x$ zu $\mathfrak{B}$ transversal macht, denn es gibt sicherlich einen zu $x$ senkrechten anisotropen Vektor $h$, und wir können $\sigma$ bei Bedarf zu $\sigma \tau(h)$ abändern. Dabei bezeichnet $\tau(h)$ die Spiegelung an $h$, also den durch

$$
\tau(h)(z)=z-q(h)^{-1} B(z, h) h
$$

definierten Automorphismus von E. Um die Existenz eines Automorphismus $\sigma$ einzusehen, der $x$ zu $\mathfrak{B}$ transversal macht, genügt es weiter nach dem Wittschen Fortsetzungssatz für Isometrien, einen Vektor $x^{\prime} \neq 0$ anzugeben, der dieselbe Länge wie $x$ hat.

Im Falle $\operatorname{dim}(E)=1$ ist nichts zu zeigen. Sei jetzt $\operatorname{dim}(E)=2$ und $\mathfrak{B}=\{e, f\}$. Es genügt, den Fall $x=\alpha e$ mit $\alpha \neq 0$ zu betrachten. Ersichtlich leistet $\sigma=\tau(f) \tau(e)$ das Gewünschte.

Sei jetzt $\operatorname{dim}(E)=3$, also insbesondere $\operatorname{Char}(A) \neq 2$. Weiter sei $A \neq \mathbb{F}_{3}$ und zunächst auch $A \neq \mathbb{F}_{5}$. Sei $\mathfrak{B}=\{e, f, g\}$ und $F$ der von $e$ und $f$ aufgespannte Teilraum von $\mathfrak{B}$. Wir wählen eine Orthogonalbasis $\left\{g_{1}, g_{2}\right\}$ von $F$. Nach Witt $[10$, S. 39] gibt es einen Vektor $\tilde{x} \neq 0$ von gleicher Länge wie $x$, der transversal zu $\left\{g_{1}, g_{2}, g\right\}$ ist. Es ist also

$$
\tilde{x}=\tilde{y}+\gamma g
$$

mit $\gamma \neq 0$ und $\tilde{y} \neq 0, \tilde{y}$ in $F$. Zu $\tilde{y}$ finden wir dann in $F$ einen Vektor $y^{\prime} \neq 0$ von gleicher Länge, der zu $\{e, f\}$ transversal ist, wie oben gezeigt wurde.

$$
x^{\prime}:=y^{\prime}+\gamma g
$$

ist dann ein Vektor der gewünschten Gestalt. Im Falle $A=\mathbb{F}_{5}$ benutzen wir ein anderes Argument. Es gibt ein $\gamma \neq 0$ in $A$, so daß $\gamma^{2} q(g)$ von $q(x)$ verschieden ist. 
Weiter gibt es in dem universellen Raum $F$ einen Vektor $\tilde{y}$ mit

$$
q(x)=q(\tilde{y})+\gamma^{2} q(g) \text {. }
$$

Man kommt nun wie zuvor zum Ziel.

Sei jetzt $\operatorname{dim}(E)=4$ und $A \neq \mathbb{F}_{2}$. Sei weiter $\mathfrak{B}=\left\{e_{1}, f_{1}, e_{2}, f_{2}\right\}$ und $F_{i}$ der Teilraum $A e_{i}+A f_{i}$ von $E$. Es genügt einen Vektor

$$
\tilde{x}=\tilde{x}_{1}+\tilde{x}_{2}
$$

mit $\tilde{x}_{i}$ in $F_{i}, \tilde{x}_{i} \neq 0$ für $i=1,2$ zu finden, der dieselbe Länge wie $x$ hat. Dann sind wir aufgrund des erledigten Falles $\operatorname{dim}(E)=2$ fertig. Ist $\operatorname{Char}(A) \neq 2$ und $A \neq \mathbb{F}_{3}, \mathbb{F}_{5}$, so ist man wieder mit der oben zitierten Bemerkung von.Witt [10, S. 39] fertig. Ist $A$ endlich, so kommt man mit dem anderen für $\operatorname{dim}(E)=3$ benutzten Argument zum Ziel: $F_{1}, F_{2}$ sind jetzt beide universell. Wir wählen einen Vektor $\tilde{x}_{2} \neq 0$ in $F_{2}$ mit $q\left(\tilde{x}_{2}\right) \neq q(z)$. Das ist möglich, weil $A \neq \mathbb{F}_{2}$ ist. Dann wählen wir einen Vektor $\tilde{x}_{1}$ in $F_{1}$ mit

$$
q(x)=q\left(\tilde{x}_{1}\right)+q\left(\tilde{x}_{2}\right) .
$$

Mit derselben Methode kommt man für $A=\mathbb{F}_{3}, \operatorname{dim} E=5$ zum Ziel.

Wir brauchen jetzt nur noch den Fall $\operatorname{dim} E=4, A$ unendlich, Char $A=2$ zu betrachten. Wir dürfen annehmen, da $\beta x$ in $F_{1}$ liegt und zu $\left\{e_{1}, f_{1}\right\}$ transversal ist. Wir suchen nun Vektoren $g$ in $F_{1}$ und $h$ in $F_{2}$, so daß $g$ nicht proportional zu $x$ ist und weiter

$$
B(x, g) \neq 0, \quad q(g)+q(h) \neq 0
$$

ist. Ist uns dies gelungen, so führt die Spiegelung an $g+h$ den Vektor $x$ in einen Vektor

$$
\tilde{x}=x-c(g+h)
$$

mit $c \neq 0$ über. Die Komponenten von $\tilde{x}$ in beiden Räumen $F_{1}, F_{2}$ sind von Null verschieden, und wir sind dann fertig. Als $g$ läßt sich nun einer der Vektoren $e_{1}, f_{1}$ wählen und als $h$ ein geeignetes skalares Vielfaches eines ausgewählten anisotropen Vektors von $F_{2}$. q.e.d.

Man sieht sofort, daß die Behauptung von Satz 2.4 stets falsch ist, wenn $\operatorname{dim} E>2$ ist und $\mathbb{F}_{2}$ als Restklassenkörper auftritt, und ebenso, wenn $\operatorname{dim} E=3$ ist und $\mathbb{F}_{3}$ als Restklassenkörper auftritt.

Ist 2 Einheit in $A$, so besitzt bekanntlich jeder quadratische Raum $E \neq 0$ über $A$ eine Orthogonalbasis, d.h. eine Basis $\left\{g_{1}, \ldots, g_{n}\right\}$ von paarweise zueinander senkrechten strikt anisotropen Vektoren $\boldsymbol{g}_{\boldsymbol{i}}$. Aufgrund der Reduktionsschritte am Anfang des soeben geführten Beweises und der schon zitierten Bemerkung $[10, \mathrm{~S} .39]$ von Witt ist auch der folgende Satz bewiesen.

Satz 2.5. Sei $2 \in A^{*}$, und jeder Restklassenkörper von $A$ enthalte mehr als 5 Elemente. Sei $\mathfrak{B}$ eine Orthogonalbasis eines quadratischen Raumes $E$ über $A$ und $x$ ein primitiver Vektor von $\mathfrak{B}$. Dann gibt es einen eigentlichen Automorphismus $\sigma$ von $E$, der $x$ in einen zu $\mathfrak{B}$ transversalen Vektor überführt.

Definition 2.6. Sei

$$
E=E_{1} \perp \cdots \perp E_{s}
$$


eine orthogonale Zerlegung eines quadratischen Raumes $E$ über $A$ in von Null verschiedene Räume $E_{i}$ und $s \geqq 2$. Ein Vektor $x$ von $E$ heiße transversal zu dieser Zerlegung, wenn

$$
x=x_{1}+\cdots+x_{s}
$$

ist mit strikt anisotropen Vektoren $x_{i}$ in $E_{i}$.

Satz 2.7. Sei eine orthogonale Zerlegung von $E$ wie in (2.6) vorgegeben, und sei $x$ ein primitiver Vektor von E. Es gelte eine der folgenden Voraussetzungen:

a) Jeder Restklassenkörper von $A$ hat mindestens 4 Elemente, und $\operatorname{dim}\left(E_{i}\right) \geqq 2$ für mindestens einen Index $i$.

b) Jeder Restklassenkörper von $A$ hat mindestens 3 Elemente, und $\operatorname{dim}\left(E_{i}\right) \geqq 2$ für mindestens zwei Indizes $i$.

c) $x$ isotrop, s gerade.

d) $x$ strikt anisotrop, $s$ ungerade.

Dann gibt es einen eigentlichen Automorphismus $\sigma$ von E, der $x$ in einen zu der Zerlegung transversalen Vektor überführt.

Beweis. Wir dürfen wieder annehmen, da $A$ ein Körper ist, und brauchen nur einen Vektor $x^{\prime}$ von gleicher Länge wie $x$ zu finden, der zu der Zerlegung transversal ist.

Wir nehmen zunächst an, daß $A$ mindestens 4 Elemente enthält. Es genügt offenbar, den Fall $s=2, \operatorname{dim} E_{1} \geqq 2, \operatorname{dim} E_{2} \geqq 1$ zu betrachten.. Sei $x=x_{1}+x_{2} \operatorname{der}$ vorgegebene primitive Vektor und $x_{i}$ in $E_{i}$. Aufgrund von Lemma 2.2 und Satz 2.4 dürfen wir $x_{1} \neq 0$ und $x_{2} \neq 0$ voraussetzen. Weiter nehmen wir an, daß mindestens einer der Vektoren $x_{i}$ isotrop ist, weil wir sonst nichts zu zeigen haben. Ist $x_{2}$ isotrop, so ist sicherlich $\operatorname{dim} E_{2} \geqq 2$. Wir dürfen also ohne Einschränkung der Allgemeinheit $q\left(x_{1}\right)=0$ voraussetzen. Wir wählen nun in $E_{2}$ einen anisotropen Vektor $y_{2}$ mit $q\left(y_{2}\right) \neq q\left(x_{2}\right)$. Das ist möglich. weil $A$ mindestens 4 Elemente enthält. Dann wählen wir in dem isotropen und somit universellen Raum $E_{1}$ einen Vektor $y_{1}$ mit

$$
q\left(y_{1}\right)=q\left(x_{2}\right)-q\left(y_{2}\right) .
$$

Der Vektor $y:=y_{1}+y_{2}$ hat dieselbe Länge wie $x$ und ist zu der Zerlegung transversal.

Sei jetzt $A=\mathbb{F}_{3}$ und $\operatorname{dim} E_{1} \geqq 2, \operatorname{dim} E_{2} \geqq 2$. Sofern $s \geqq 3$ ist, wählen wir in jedem Raum $E_{i}$ mit $i \geqq 3$ einen anisotropen Vektor $y_{i}$. Weil die Räume $E_{1}$ und $E_{2}$ beide universell sind, gibt es nun anisotrope Vektoren $y_{1}$ in $E_{1}$ und $y_{2}$ in $E_{2}$, so daß

$$
q(x)=q\left(y_{1}\right)+q\left(y_{2}\right)+\cdots+q\left(y_{s}\right)
$$

im Falle $s \geqq 3$ und

$$
q(x)=q\left(y_{1}\right)+q\left(y_{2}\right)
$$

im Falle $s=2$ ist. Der Vektor

$$
y:=y_{1}+\cdots+y_{s}
$$

ist transversal zu der Zerlegung und hat dieselbe Länge wie $x$. 
Sei schließlich $A=\mathbb{F}_{2}$. Wir wählen in jedem Raum $E_{i}$ einen anisotropen Vektor $y_{i}$. Dann hat

$$
y:=y_{1}+\cdots+y_{s}
$$

dieselbe Länge wie $x$, falls entweder $x$ isotrop und $s$ gerade oder $x$ anisotrop und $s$ ungerade ist. Damit ist der Beweis von Satz 2.7 beendet.

Mit Hilfe des soeben bewiesenen Satzes läßt sich der frühere Transversalitätssatz 2.4 wie folgt ausweiten.

Satz 2.8. Jeder Restklassenkörper von $A$ enthalte mindestens 4 Elemente. $E$ sei ein quadratischer Raum über $A$ und $\mathfrak{B}$ sei eine kanonische Basis von $E$.

i) Ist $\operatorname{dim}(E) \geqq 3$, so ergibt es zu jedem primitiven Vektor $x$ von $E$ einen eigentlichen Automorphismus $\sigma$ von $E$, so daß $\sigma(x)$ transversal zu $\mathfrak{B}$ ist.

ii) Ist $\operatorname{dim}(E)=2$, so gilt dies wenigstens für strikt anisotrope Vektoren $x$.

Beweis. Aufgrund von Satz 2.7 mit der Voraussetzung a) genügt es, die zweite Behauptung zu beweisen. Wir dürfen $A$ wieder als Körper voraussetzen. Sei $\mathfrak{B}=\{e, f\}$. Sind $q(e)$ und $q(f)$ beide $\neq 0$, so sind wir aufgrund von Satz 2.4 fertig. Sei jetzt $q(e)=a, q(f)=0$, wobei für $a$ der Wert Null zugelassen ist. Es genügt, einen zu $\mathfrak{B}$ transversalen Vektor $y$ gleicher Länge wie $x$ anzugeben. Denn dann gibt es einen Automorphismus $\rho$ von $E$ mit $\rho(x)=y$, und $\sigma=\rho$ oder $\sigma=\rho \tau(x)$ leistet das Verlangte. Wir machen den Ansatz

und haben die Gleichung

$$
y=\lambda e+\mu f
$$

$$
\lambda^{2} a+\lambda \mu=q(x)
$$

mit $\lambda \neq 0, \mu \neq 0$ zu lösen. Dazu wählen wir zunächst ein $\lambda \neq 0$ mit $\lambda^{2} a \neq q(x)$. Das ist möglich, weil $q(x) \neq 0$ ist und $A$ mindestens 4 Elemente enthält. Dann lösen wir obige Gleichung nach $\mu$ auf. q.e.d.

\section{§ 3. Erzeugnis des Annulators durch binäre und quaternäre Räume}

Sei zunächst $A$ ein beliebiger semilokaler Ring und $\varphi$ ein binärer bilinearer Pfisterraum $(1, a)$ über $A$. Der folgende Satz wurde über Körpern einer Charakteristik $\neq 2$ von Elman und Lam bemerkt [5, Prop. 2.2] und als nützliches Hilfsmittel vielfältig angewendet.

Satz 3.1. Jeder quadratische Raum $E$ über A besitzt eine Zerlegung

$$
E=E_{1} \perp \cdots \perp E_{r} \perp G
$$

mit binären Räumen $E_{i}$, für die $\varphi \otimes E_{i}$ hyperbolisch ist, und einem Raum $G$, für den $\varphi \otimes G$ anisotrop oder $\operatorname{dim} G=1$ ist. $\{r=0$, falls $\varphi \otimes E$ anisotrop. $\}$

Für einen beliebigen Bilinearraum $\psi$ über $A$ bezeichnen wir den Wert der Bilinearform auf einem Vektorpaar $x, y$ gewöhnlich mit $(x, y)$, und für den Wert $(x, x)$ schreiben wir auch $n(x)$. Satz 3.1 ergibt sich sofort aus der folgenden Transversalitätsaussage. 
Lemma 3.2. Sei $\operatorname{dim} E \geqq 2$ und $\varphi \otimes E$ isotrop. Weiter sei $\left\{g_{1}, g_{2}\right\}$ eine Orthogonalbasis von $\varphi$ mit $n\left(g_{1}\right)=1, n\left(g_{2}\right)=a$. Schließlich sei $z$ ein primitiver isotroper Vektor von $\varphi \otimes E$. Dann gibt es einen eigentlichen Automorphismus $\sigma$ von $\varphi \otimes E$, so daß $\sigma(z)$ die Gestalt $g_{1} \otimes x+g_{2} \otimes y$ hat mit Vektoren $x, y$ aus $E$, die einen binären nicht entarteten Raum $A x+A y$ aufspannen.

In der Tat, angenommen Lemma 3.2 sei schon bewiesen. Zum Beweis von Satz 3.1 genügt es offenbar einzusehen, daß sich von $E$ ein binärer Raum $F$ mit $\varphi \otimes F$ hyperbolisch abspalten läßt, falls $\operatorname{dim} E \geqq 2$ und $\varphi \otimes E$ isotrop ist. Ein solcher Raum ist nun der Raum $F=A x+A y$ aus dem Lemma 3.2, denn $\varphi \otimes F$ ist isotrop und somit nach $[1, \S 1]$ hyperbolisch.

Wir kommen jetzt zu dem Beweis des Lemmas 3.2 und durchlaufen zunächst dieselben Reduktionsschritte wie zu Anfang des Beweises von Satz 2.4. Wir nehmen also an, daß $A$ ein Körper ist, und wir brauchen nur in dem isotropen $\operatorname{Raum} \varphi \otimes E$ einen isotropen Vektor

$$
z^{\prime}=g_{1} \otimes x+g_{2} \otimes y
$$

anzugeben, dessen Komponenten $x$ und $y$ einen nicht entarteten binären Raum aufspannen. Das bedeutet, daß wir Vektoren $x, y$ in $E$ finden müssen, die einen nicht entarteten binären Teilraum aufspannen und der Gleichung

genügen.

$$
q(x)+a q(y)=0
$$

Ist $E$ schon isotrop, so wählen wir $x$ und $y$ als ein hyperbolisches Vektorpaar in $E$ dd.h. $q(x)=q(y)=0, B(x, y)=1\}$. Ist $a=-1$, so nehmen wir einen nicht entarteten binären Teilraum $F$ von $E$ her, und wählen darin eine kanonische Basis $\{e, f\}$ mit $q(e) \neq 0$. Dann leisten $x=f$ und $y=\tau(e) f$ das Verlangte.

Ab jetzt seien $E$ und $\varphi$ beide anisotrop. Sei $g_{1} \otimes u+g_{2} \otimes v$ ein isotroper Vektor $\neq 0$ in $\varphi \otimes E$. Es ist dann

$$
q(u)+a q(v)=0
$$

und wegen der Anisotropie von $E$ müssen $q(u)$ und $q(v)$ beide von Null verschieden sein. Wir führen die Bezeichnungen

$$
q(v)=\lambda, \quad B(u, v)=\mu
$$

ein und haben $q(u)=-a \lambda$. Ist $\mu^{2}+4 a \lambda^{2} \neq 0$, so leisten $x=u$ und $y=v$ das Verlangte. Ab jetzt sei $\mu^{2}+4 a \lambda^{2}=0$. Weil $-a$ kein Quadrat in $A$ ist, muß der Körper $A$ die Charakteristik 2 haben und weiter $\mu=0$ sein. $E$ hat also eine Dimension $\geqq 4$ und enthält Vektoren $r, s$, so daß die Wertetabelle der quadratischen Form für die Folge von Vektoren $u, r, v, s$ die Gestalt

$$
\left[\begin{array}{cc}
-a \lambda & 1 \\
1 & b
\end{array}\right] \perp\left[\begin{array}{ll}
\lambda & 1 \\
1 & c
\end{array}\right]
$$

mit $b, c$ in $A$ hat. Der Vektor $r+s$ ist anisotrop, weil von Null verschieden, und der Vektor

$$
y:=\tau(r+s)(v)
$$


hat dieselbe Länge wie $v$ und steht nicht auf $u$ senkrecht. Somit leisten die Vektoren $x=u$ und $y$ das Verlangte. Damit ist Lemma 3.2 und Satz 3.1 vollständig bewiesen.

Für einen bilinearen Raum $\psi$ über $A$ bezeichnen wir mit $\mathfrak{U}(\psi)$ den $W(A)$-Modul, der von den Klassen aller quadratischen Räume $E$ in $W q(A)$ gebildet wird, für die $\psi \otimes \mathrm{E} \sim 0$ und $\operatorname{dim} E$ gerade ist. $\mathfrak{U}(\psi)$ ist der volle Annullator von $\psi$ in $W q(A)$ außer in dem uninteressanten Falle, daß 2 Einheit und $\psi$ hyperbolisch ist.

Als eine Abschwächung von Satz 3.1 erhalten wir

Korollar 3.3. Sei $\varphi$ ein binärer bilinearer Pfisterraum. Dann wird $\mathfrak{A}(\varphi)$ als $W(A)$ Modul von den Räumen $[1, b]$ mit $\varphi \otimes[1, b] \sim 0$ erzeugt.

Für einen beliebigen bilinearen Pfisterraum $\varphi=\left(1, a_{1}\right) \otimes \cdots \otimes\left(1, a_{n}\right)_{0}$ können wir zunächst nur das folgende schwächere Resultat beweisen.

Satz 3.4. A sei beliebiger semilokaler Ring. Dann wird $\mathfrak{U}(\varphi)$ als $W(A)$-Modul von folgenden Räumen erzeugt.

1. Räume $[1, b]$ mit $\varphi \otimes[1, b] \sim 0$

2. Räume $(1,-a) \otimes[1, b]$ mit a eine von $\varphi \otimes[1, b]$ dargestellte Einheit.

Wir wollen diese Räume als Räume vom Typ 1 bzw. vom Typ 2 zu $\varphi$ bezeichnen. Zum Beweis von Satz 3.4 sei zunächst angemerkt, daß die Räume vom Typ 2 tatsächlich in $\mathfrak{U}(\varphi)$ liegen. Ist nämlich $a$ eine von $\varphi \otimes[1, b]$ dargestellte Einheit, so ist $a$ Ähnlichkeitsnorm dieses Raumes und somit $(1,-a) \otimes \varphi \otimes[1, b]$ hyperbolisch.

Sei $\mathfrak{B}(\varphi)$ der von den Räumen vom Typ 1 und $2 \mathrm{zu} \varphi$ erzeugte Untermodul von $\mathfrak{U}(\varphi)$, und sei $E$ ein quadratischer Raum gerader Dimension $2 r$ mit $\varphi \otimes E \sim 0$. Wir wollen durch Induktion nach $r$ zeigen, daß $E$ in $\mathfrak{B}(\varphi)$ liegt. Dabei setzen wir aufgrund von Korollar $3.3 \operatorname{dim}(\varphi)>2$ voraus.

Im Falle $r=1$ ist nichts zu zeigen. Sei jetzt $r>1$ und

$$
E \cong\left(a_{1}\right) \otimes\left[1, b_{1}\right] \perp \cdots \perp\left(a_{r}\right) \otimes\left[1, b_{r}\right]
$$

eine Zerlegung von $E$ in binäre Räume. Zunächst nehmen wir an, daß $r$ gerade ist. Der Raum $\varphi \otimes E$ ist isotrop. Nach Satz 2.7 mit der Voraussetzung c) gibt es strikt anisotrope Vektoren $x_{i}$ in den Räumen $\varphi \otimes\left[1, b_{i}\right]$ mit

$$
a_{1} q\left(x_{1}\right)+\cdots+a_{r} q\left(x_{r}\right)=0 .
$$

Wir betrachten nun den Raum

$$
F:=\left(a_{1} q\left(x_{1}\right)\right) \otimes\left[1, b_{1}\right] \perp \cdots \perp\left(a_{r} q\left(x_{r}\right)\right) \otimes\left[1, b_{r}\right] .
$$

Dieser Raum ist isotrop und es gilt

mit Räumen

$$
E \sim\left(a_{1}\right) \otimes G_{1} \perp \cdots \perp\left(a_{r}\right) \otimes G_{r} \perp F .
$$

$$
G_{i}:=\left(1,-q\left(x_{i}\right)\right) \otimes\left[1, b_{i}\right],
$$

die vom Typ 2 sind. $F$ liegt also wieder in $\mathfrak{A}(\varphi)$ und es ist $F \sim G$ mit einem Raum $G$ einer Dimension $<2 r$. Nach Induktionsvoraussetzung liegt $G$ in $\mathfrak{B}(\varphi)$ und damit auch $F$ und $E$.

4 Math. Z., Bd. 140 
Sei jetzt $r$ ungerade. Wir bezeichnen mit $E^{\prime}$ die orthogonale Summe der Räume $\left(a_{i}\right) \otimes\left[1, b_{i}\right]$ für $1 \leqq i \leqq r-1$. Ersichtlich ist

$$
\varphi \otimes E^{\prime} \sim\left(-a_{r}\right) \otimes \varphi \otimes\left[1, b_{r}\right] .
$$

Weil $r \geqq 3$ ist, hat die rechte Seite kleinere Dimension als die linke Seite, und $\varphi \otimes E^{\prime}$ ist also isotrop. Nach Satz 2.7 gibt es strikt anisotrope Vektoren $x_{i}$ in den Räumen $\varphi \otimes\left[1, b_{i}\right]$ für $1 \leqq i \leqq r-1$, so daß

$$
a_{1} q\left(x_{1}\right)+\cdots+a_{r-1} q\left(x_{r-1}\right)=0
$$

ist. Man kommt nun wie zuvor zum Ziel, indem man den Raum

$$
F:=\left(a_{1} q\left(x_{1}\right)\right) \otimes\left[1, b_{1}\right] \perp \cdots \perp\left(a_{r-1} q\left(x_{r-1}\right)\right) \otimes\left[1, b_{r-1}\right] \perp\left(a_{r}\right) \otimes\left[1, b_{r}\right]
$$

betrachtet. Damit ist Satz 3.4 bewiesen.

Wir schließen diesen Paragraphen mit einer Anwendung von Satz 3.4. Zunächst sei an einige wohlbekannte Begriffe erinnert [vgl. 3, 9]. $\mathrm{Zu}$ einem quadratischen Raum $E$ über $A$ bezeichne $D(E)$ die Diskriminantenalgebra von $E$. Diese ist in dem hier alleine interessierenden Fall, daß $E$ gerade Dimension hat, definiert als das Zentrum der 2. Cliffordalgebra $C^{+}(E)$. Sie ist eine quadratische etale $\mathrm{Er}$ weiterung, also von der Gestalt $A \oplus A \omega$ mit einer Relation $\omega^{2}-\omega=b$, wobei $b \in A$ und $1+4 b \in A^{*}$ ist. Eine solche Erzeugende $\omega$ bezeichnen wir mit $\frac{1}{\wp} b$. Eine quadratische etale Erweiterung $B=A\left[\frac{1}{\wp} b\right]$ ist unter ihrer Normform ein quadratischer Raum, den wir mit $[B]$ bezeichnen. Es ist $[B] \cong[1,-b]$ und $D([B]) \cong B$.

Satz 3.5. Sei $A$ beliebiger semilokaler Ring. Sei $\varphi$ ein bilinearer Pfisterraum und $E$ ein quadratischer Raum über $A$ mit $\varphi \otimes E \sim 0$. Dann ist auch $\varphi \otimes[D(E)] \sim 0$.

Beweis. Wir dürfen $\varphi$ als anisotrop voraussetzen. $E$ hat dann gerade Dimension. Nach Satz 3.4 ist

$$
E \sim\left(a_{1}\right) \otimes F_{1} \perp \cdots \perp\left(a_{s}\right) \otimes F_{s} \perp\left(c_{1}\right) \otimes\left[B_{1}\right] \perp \cdots \perp\left(c_{r}\right) \otimes\left[B_{r}\right]
$$

mit Räumen $F_{i}$ vom Typ 2 zu $\varphi$ und quadratischen etalen Algebren $B_{i}$, die Räume $\left[B_{i}\right]$ vom Typ 1 liefern. Es sei daran erinnert, daß wir für quadratische etale Erweiterungen $B=A\left[\frac{1}{\wp} b\right]$ und $B^{\prime}=A\left[\frac{1}{\wp} b^{\prime}\right]$ eine wohldefinierte Komposition

$$
B \circ B^{\prime}=A\left[\frac{1}{\wp}\left(b+b^{\prime}+4 b b^{\prime}\right)\right]
$$

haben [3, S. 128]. Damit berechnet man

$$
D(E) \cong B_{1} \circ \cdots \circ B_{t} .
$$

Nun ist für zwei quadratische etale Erweiterungen $B$ und $B^{\prime}$

$$
[B] \perp(-1) \otimes\left[B^{\prime}\right] \cong[0,0] \perp(a) \otimes\left[B \circ B^{\prime}\right]
$$


mit einer Einheit $a$ von $A$, denn die linke Seite ist isotrop und hat die Diskriminante $B \circ B^{\prime}$. Aus dieser Bemerkung und der Tatsache, daß die Räume $\varphi \otimes\left[B_{i}\right]$ hyperbolisch sind, folgt, daß $\varphi \otimes[D(E)]$ hyperbolisch ist. q.e.d.

\section{§4. Eine Erweiterung des Transversalitätssatzes 2.8}

Sei $\psi$ ein eigentlicher bilinearer Raum einer Dimension $\geqq 2$ und $E$ ein binärer quadratischer Raum über $A$. Weiter sei $\{e, f\}$ eine kanonische Basis von $E$ und $z$ ein primitiver Vektor des Raumes $\psi \otimes E$. Wir wollen das folgende Lemma beweisen.

Lemma 4.1. Es gelte eine der folgenden Voraussetzungen:

a) $\operatorname{dim} \psi \geqq 3$;

b) $\operatorname{dim} \psi=2$, und $A$ hat $\mathbb{F}_{3}$ nicht als Restklassenkörper;

c) $\operatorname{dim} \psi=2$, und $z$ ist strikt anisotrop.

Dann gibt es einen eigentlichen Automorphismus $\sigma$ von $\psi \otimes E$, der $z$ in einen Vektor $x^{\prime} \otimes e+y^{\prime} \otimes f$ überführt mit strikt anisotropen Vektoren $x^{\prime}$ und $y^{\prime}$ aus $\psi$ $\left\{\right.$ d.h. $n\left(x^{\prime}\right)$ und $n\left(y^{\prime}\right)$ sind Einheiten $\}$.

Beweis. Wir dürfen annehmen, daß $A$ ein Körper ist, und brauchen nur einen Vektor $z^{\prime}=x^{\prime} \otimes e+y^{\prime} \otimes f$ anzugeben mit $n\left(x^{\prime}\right) \neq 0, n\left(y^{\prime}\right) \neq 0$ und $q\left(z^{\prime}\right)=q(z)$, vgl. Anfang des Beweises von Satz 2.4.

Wir behandeln zunächst den Fall $q(f)=0$. Wir wählen in $\psi$ zwei zueinander senkrechte anisotrope Vektoren $u, v$ und machen den Ansatz

$$
x^{\prime}=u, \quad y^{\prime}=\lambda u+\mu v .
$$

Dann haben wir an $\lambda$ und $\mu$ in $A$ die folgenden beiden Bedingungen zu stellen:

$$
\begin{gathered}
\lambda^{2} n(u)+\mu^{2} n(v) \neq 0 . \\
n(u) q(e)+\lambda n(u)=q(z) .
\end{gathered}
$$

Diese Bedingungen lassen sich für jeden Körper $A$ simultan erfüllen.

Ab jetzt sei $q(e) \neq 0, q(f) \neq 0$. Sei

$$
z=x \otimes e+y \otimes f .
$$

Aufgrund von Satz 2.4 dürfen wir, außer in dem Falle $A=\mathbb{F}_{2}$, voraussetzen, $\mathrm{da} \beta x \neq 0$ und $y \neq 0$ ist. \{Wir könnten sogar annehmen, da $\beta x$ und $y$ transversal zu einer vorgegebenen Orthogonalbasis von $\psi$ sind.\} Weiter nehmen wir an, $\mathrm{da} ß x$ und $y$ nicht schon beide anisotrop sind, und dann ohne Einschränkung der Allgemeinheit $n(x)=0$. Indem wir schließlich die quadratische Form von $E$ und den Vektor $f$ mit geeigneten Skalaren multiplizieren, erreichen wir, daß zusätzlich $q(e)=1$ ist. Den Wert $q(f)$ bezeichnen wir mit $b$.

Wir behandeln zunächst 4 besonders einfache Fälle (i)-(iv). Ist Char $A=2$ und $A$ endlich, so werden wir dabei unsere Voraussetzung $x \neq 0, y \neq 0$ nicht benutzen. Somit ist jetzt auch der Körper $A=\mathbb{F}_{2}$ zugelassen.

(i) Char $A \neq 2, q(z) \neq 0$

(ii) Char $A=2, A$ endlich, $q(z) \neq 0$. 
Im ersten Falle ist der Raum $\psi$ universell, weil $\psi$ isotrop ist. Auch im zweiten Falle ist $\psi$ universell, denn dann ist jedes Element von $A$ ein Quadrat. Wir wählen nun einen Vektor $u$ in $\psi$ mit

Dann ist

$$
n(u)=b^{-1} q(z)
$$

$$
z^{\prime}=u \otimes e-u \otimes f
$$

ein Vektor der gewünschten Gestalt.

(iii) $b$ ist Quadrat in $A, q(z)=0$.

Der Raum $\psi$ enthält zueinander senkrechte anisotrope Vektoren $u, v$ mit $n(v)=-n(u)$. Sei $b=\lambda^{2}$ mit $\lambda$ in $A$. Der Vektor

$$
z^{\prime}=\lambda u \otimes e+v \otimes f
$$

hat dann anisotrope Komponenten und die Länge Null.

Damit haben wir insbesondere den Fall, daß $A$ endlich und $\operatorname{Char}(A)=2$ ist, völlig erledigt.

(iv) Char $A \neq 2, \operatorname{dim} \psi>2, q(z)=0$.

Wir ergänzen $x$ zu einem hyperbolischen Vektorpaar $x, u$ und wählen einen zu $x$ und $u$ senkrechten anisotropen Vektor $w$. Dann machen wir den Ansatz.

$$
z^{\prime}=(u+\lambda x) \otimes e+w \otimes f
$$

und haben für $\lambda$ die Bedingungen

$$
\lambda \neq 0, \quad 2 \lambda+n(w) b=0
$$

zu stellen, die sich erfüllen lassen.

Nachdem wir diese Fälle (i) - (iv) vorweg erledigt haben, gehen wir jetzt so vor, daß wir $z$ direkt durch Anwendung von geeigneten Spiegelungen in die gewünschte Gestalt überführen. Wir diskutieren getrennt die Fälle $n(y) \neq 0$ und $n(y)=0$.

Sei zunächst $n(y) \neq 0$. Durch Anwendung von $\tau(y \otimes e)$ auf $z$ erhalten wir den Vektor

$$
z^{\prime}=x^{\prime} \otimes e+y \otimes f
$$

mit

$$
x^{\prime}=x-[2(x, y)+n(y)] n(y)^{-1} y .
$$

Man errechnet

$$
n\left(x^{\prime}\right)=2(x, y)+n(y)
$$

Somit sind wir fertig, falls $2(x, y)+n(y) \neq 0$ ist. Sei jetzt $2(x, y)=-n(y)$ und insbesondere Char $A \neq 2$. Indem wir die Bilinearform von $\psi$ um einen skalaren Faktor abändern, können wir $(x, y)=1, n(y)=-2$ annehmen. Wir wenden auf $z$ eine Spiegelung $\tau(g)$ mit

$$
g=(\lambda x+y) \otimes e
$$


für geeignetes $\lambda \in A$ an. Es ist

$$
q(g)=2(\lambda-1), \quad B(g, z)=\lambda .
$$

Wir müssen also zunächst $\lambda \neq 0, \lambda \neq 1$ fordern. Es ist dann

mit

$$
\tau(g)(z)=x^{\prime} \otimes e+y \otimes f
$$

und

$$
x^{\prime}=-[2(\lambda-1)]^{-1}\left[\left(\lambda^{2}-2 \lambda+2\right) x+\lambda y\right],
$$

$$
2 n\left(x^{\prime}\right)=\lambda(\lambda-2)(\lambda-1)^{-1} \text {. }
$$

Wir müssen also auch $\lambda \neq 2$ fordern. Diese Forderungen sind für $A \neq \mathbb{F}_{3}$ erfüllbar.

Sei jetzt $n(x)=n(y)=0$. Wir betrachten zunächst den Fall $(x, y) \neq 0$ und nehmen wieder $(x, y)=1$ an. Es ist also auch $q(z)=1$, und nach (i) dürfen wir Char $A=2$ voraussetzen. Der Raum $A x+A y$ ist nicht ausgeartet und nicht eigentlich. Also ist $\operatorname{dim} \psi>2$ und es gibt einen zu $x$ und $y$ senkrechten anisotropen Vektor $v$ in $\psi$. Sei $n(v)=c$. Wir betrachten

$$
g=(y+v) \otimes f
$$

Es ist $q(g)=c b$ und $B(z, g)=1$, und wir erhalten

mit

$$
\tau(g)(z)=x \otimes e+y^{\prime} \otimes f
$$

$$
b c y^{\prime}=(b c-1) y-v \text {. }
$$

Man sieht, daß $y^{\prime}$ anisotrop ist, und wir haben uns auf den schon betrachteten Fall $n(x)=0, n(y) \neq 0$ zurückgezogen.

Sei jetzt $(x, y)=0$, aber $y$ nicht zu $x$ proportional. Wegen (iv) dürfen wir wieder Char $A=2$ annehmen. Indem wir eventuell die Rolle von $x$ und $y$ und die Rolle von $e$ und $f$ vertauschen, können wir annehmen, daß es einen anisotropen Vektor $u$ in $\psi$ gibt mit $(u, x)=1$ und $(u, y)=0$. Für $g=u \otimes f$ erhalten wir

mit

$$
\tau(g)(z)=x \otimes e+y^{\prime} \otimes f
$$

$$
y^{\prime}=y-n(u)^{-1} b^{-1} u,
$$

also $n\left(y^{\prime}\right) \neq 0$, und sind damit bei einem erledigten Fall angekommen.

Sei schließlich $y=\alpha x, \alpha \neq 0$. Wir wählen einen anisotropen Vektor $u$ in $\psi$ mit $(u, x) \neq 0$. Indem wir die Form von $\psi$ und den Vektor $z$ mit geeigneten Skalaren multiplizieren, können wir

$$
n(u)=(u, x)=1
$$

erreichen. Für $g:=u \otimes e$ erhalten wir

$$
\tau(g)(z)=[x-(2+\alpha) u] \otimes e+\alpha x \otimes f .
$$

Ist also $\alpha \neq-2$, so haben wir uns auf den Fall $(x, y) \neq 0$ zurückgezogen. Sei jetzt $\alpha=-2$ und insbesondere Char $A \neq 2$. Dann wählen wir $g=u \otimes(e-f)$ und erhalten

$$
q(g)=b, \quad B(z, g)=4 b-1 \neq 0,
$$


also mit $\lambda:=(4 b-1) b^{-1}$

$$
\tau(g)(z)=(x-\lambda u) \otimes e+(-2 x+\lambda u) \otimes f
$$

Wir sind damit bei dem schon erledigten Fall, daß $x$ und $y$ nicht proportional sind, angelangt.

Jetzt ist Lemma 4.1 völlig bewiesen. Nur der folgende Fall blieb bei dieser Betrachtung offen:

$$
A=\mathbb{F}_{3}, \quad \psi \cong(1,-1), \quad q(e)=1, \quad q(f)=-1, \quad q(z)=0 .
$$

In diesem Falle müssen wir anisotrope Vektoren $x^{\prime}$ und $y^{\prime}$ in $\psi$ finden, die der Gleichung

$$
n\left(x^{\prime}\right)+\left(x^{\prime}, y^{\prime}\right)-n\left(y^{\prime}\right)=0
$$

genügen. Man überzeugt sich leicht, daß es solche Vektoren $x^{\prime}$ und $y^{\prime}$ nicht gibt.

Wir können jetzt einen Satz beweisen, der sich als Erweiterung des Transversalitätssatzes 2.8 ansehen läßt.

Satz 4.2. Sei $\left\{z_{1}, \ldots, z_{n}\right\}$ eine kanonische Basis eines quadratischen Raumes $E$ einer Dimension $n \geqq 2$, und sei $\psi$ ein eigentlicher bilinearer Raum einer Dimension $\geqq 2$ über $A$. Im Falle $\operatorname{dim} E=\operatorname{dim} \psi=2$ sei vorausgesetzt, daß $A$ nicht $\mathbb{F}_{3}$ als Restklassenkörper hat. Dann läßt sich jeder primitive Vektor $z$ von $\psi \otimes E$ durch einen eigentlichen Automorphismus $\sigma$ von $\psi \otimes E$ in die Gestalt

$$
\sigma(z)=u_{1} \otimes z_{1}+\cdots+u_{n} \otimes z_{n}
$$

mit strikt anisotropen Vektoren $u_{i}$ aus $\psi$ überführen.

Beweis. Ist $\operatorname{dim}(E)=2$, so wird unsere Behauptung durch Lemma 4.1 gedeckt. Sei jetzt $\operatorname{dim}(E) \geqq 3$ und

$$
E=E_{1} \perp \cdots \perp E_{s}
$$

die zu der kanonischen Basis gehörige Zerlegung von $E$ in Räume der Dimension 1 oder 2, wobei höchstens $E_{s}$ die Dimension 1 hat. Wir dürfen wieder voraussetzen, daß $A$ ein Körper ist. Ist $A \neq \mathbb{F}_{2}$, so können wir $z$ aufgrund von Satz 2.7 in einen Vektor

$$
\tilde{z}:=x_{1}+\cdots+x_{s}
$$

mit anisotropen $x_{i}$ in $\psi \otimes E_{i}$ transformieren und dann Lemma 4.1 mit der Voraussetzung a) oder c) auf $x_{1}, \ldots, x_{s-1}$ und gegebenenfalls $x_{s}$ anwenden. Sei jetzt $A=\mathbb{F}_{2}$. Die Räume $\psi \otimes E_{i}$ sind sicherlich isotrop. Daher können wir Vektoren $x_{i} \neq 0$ in $\psi \otimes E_{i}$ finden, so daß

$$
q(z)=q\left(x_{1}\right)+\cdots+q\left(x_{s}\right)
$$

ist. $z$ läßt sich in den Vektor

$$
\tilde{z}=x_{1}+\cdots+x_{s}
$$

transformieren. Man wende dann Lemma 4.1 mit der Voraussetzung a) oder b) auf die Vektoren $x_{1}, \ldots, x_{s}$ an. q.e.d.

Im folgenden Paragraphen benötigen wir zunächst nur eine Abschwächung der Aussage von Lemma 4.1, die über jedem semilokalen Ring gilt. Wir betrachten wieder die vor Lemma 4.1 beschriebene Situation. 
Lemma 4.3. Es gibt stets einen eigentlichen Automorphismus $\sigma$ von $\psi \otimes E$ der $z$ in einen Vektor $x^{\prime} \otimes e+y^{\prime} \otimes f$ mit strikt anisotropem $y^{\prime}$ überführt.

Beweis. Aufgrund des Beweises von Lemma 4.1 brauchen wir dies nur noch in der vor Satz 4.2 beschriebenen Situation (*) zu zeigen. Man kann dann die Gleichung (**) mit anisotropem $y^{\prime}$ und isotropem $x^{\prime}$ lösen. q.e.d.

\section{§5. Beschreibung der Räume vom Typ 1}

Wie früher bezeichne $\varphi$ einen bilinearen Pfisterraum einer Dimension $>1$. Wir wählen in $\varphi$ einen Vektor $\varepsilon$ der Länge 1 fest aus und haben eine Zerlegung

$$
\varphi=A \varepsilon \perp \varphi^{\prime}
$$

Wir wollen die Räume vom Typ 1 zu $\varphi$ beschreiben, also die binären Räume $[1, b]$ mit $\varphi \otimes[1, b] \sim 0$. Wir geben zunächst Beispiele solcher Räume. Sei $b$ ein Element aus $A$ der Gestalt

$$
b=-\gamma-\gamma^{2}-n(v)
$$

mit $\gamma$ in $A$ und $v$ in $\varphi^{\prime}$. Weiter sei $1-4 b$ Einheit. Dann wird der Raum $E:=[1, b]$ von $\varphi$ annuliert. In der Tat, $E$ stellt das Element $\gamma^{2}+\gamma+b$ primitiv dar und $\varphi^{\prime} \otimes E$ stellt das Element $n(v)$ dar. Somit ist

$$
\varphi \otimes E \cong E \perp \varphi^{\prime} \otimes E
$$

isotrop, also nach $[1, \S 1]$ hyperbolisch.

Satz 5.1. A sei beliebig semilokal. Dann gibt es zu jedem Raum E vom Typ 1 zu $\varphi$ Elemente $\gamma$ in $A$ und $v$ in $\varphi^{\prime}$, so daß

$$
E \cong\left[1,-\gamma-\gamma^{2}-n(v)\right] .
$$

Beweis. Sei $E=[1, b]$ und $\varphi \otimes E$ hyperbolisch. Nach Lemma 4.3 gibt es Vektoren $x, y$ in $\varphi$, so daß $n(y)$ Einheit und

$$
n(x)+(x, y)+n(y) b=0
$$

ist. Sei $z$ der Vektor $n(y)^{-1} y$. Wir erhalten die Gleichung

$$
b=-n(x) n(z)-(x, z) \text {. }
$$

Nach [2] gibt es einen Vektor $w$ in $\varphi$, so daß

und

$$
n(x) n(z)=n(w)
$$

mit $v \in \varphi^{\prime}$ ist. Wir definieren

$$
w=(x, z) \varepsilon+v
$$

und erhalten

$$
\gamma:=(x, z)=(x, y) n(y)^{-1}
$$

$$
b=-\gamma-\gamma^{2}-n(v) \text {. q.e.d. }
$$

Wenn $A$ nicht zu kleine Restklassenkörper hat, läßt sich unser Resultat noch verbessern. 
Satz 5.2. Es gelte eine der beiden folgenden Voraussetzungen:

a) $\operatorname{dim} \varphi>2$;

b) $\operatorname{dim} \varphi=2, A$ hat $\mathbb{F}_{3}$ nicht als Restklassenkörper.

Dann hat jeder Raum E vom Typ 1 zu $\varphi$ die Gestalt

$$
E \cong[1,-n(v)]
$$

mit $v$ in $\varphi^{\prime}$. Dabei läßt sich $v$ sogar als strikt anisotroper Vektor von $\varphi^{\prime}$ wählen, wenn eine der beiden folgenden stärkeren Voraussetzungen erfüllt ist.

c) $\operatorname{dim} \varphi>2, A$ hat weder $\mathbb{F}_{2}$ noch $\mathbb{F}_{3}$ als Restklassenkörper;

d) $\operatorname{dim} \varphi=2$, A hat keinen der Körper $\mathbb{F}_{2}, \mathbb{F}_{3}, \mathbb{F}_{5}$ als Restklassenkörper.

Beweis. Wir schließen an den Beweis von Satz 5.1 an. Sei $e, f$ eine kanonische Basis von $E$ mit

$$
q(e)=1, \quad q(f)=b=-\gamma-\gamma^{2}-n(v) .
$$

Ist nun $1+2 \gamma$ Einheit, so ist

kanonische Basis mit

$$
e, f^{\prime}:=(1+2 \gamma)^{-1}(\gamma e+f)
$$

Man berechnet

$$
q(e)=1, \quad q(f)=-(1+2 \gamma)^{-2} n(v) .
$$

und

$$
1+2 \gamma=n(y)^{-1}[n(y)+2(x, y)]
$$

$$
n(v)=n(y)^{-2}\left[n(x) n(y)-(x, y)^{2}\right] .
$$

Wir müssen unsere Vektoren $x, y$ in $\varphi$ also so wählen, daß neben den Bedingungen

$$
n(y) \in A^{*}, \quad n(x)+(x, y)+n(y) b=0
$$

auch die Bedingungen

und möglichst

$$
n(y)+2(x, y) \in A^{*}
$$

$$
n(x) n(y)-(x, y)^{2} \in A^{*}
$$

gelten. Somit ist der Beweis von Satz 5.2 erbracht, wenn die folgende Verfeinerung des früheren Lemmas 4.3 gezeigt ist.

Lemma 5.3. Wir betrachten die zu Anfang von $\$ 4$ beschriebene Situation und setzen jetzt $q(e)=1$ und $q(z)=0$ voraus.

i) Es gelte eine der beiden folgenden Voraussetzungen:

a) $\operatorname{dim} \psi \geqq 3$;

b) $\operatorname{dim} \psi=2$, A hat $\mathbb{F}_{3}$ nicht als Restklassenkörper.

Dann gibt es einen eigentlichen Automorphismus $\sigma$ von $\psi \otimes E$, so daß

$$
\sigma(z)=x^{\prime} \otimes e+y^{\prime} \otimes f
$$

ist mit Vektoren $x^{\prime}, y^{\prime}$ aus $\psi$, für die $n\left(y^{\prime}\right)$ und $2\left(x^{\prime}, y^{\prime}\right)+n\left(y^{\prime}\right)$ Einheiten sind. 
ii) Man kann zusätzlich erreichen, daß $n\left(x^{\prime}\right) n\left(y^{\prime}\right)-\left(x^{\prime}, y^{\prime}\right)^{2}$ Einheit ist, also daß $x^{\prime}$ und $y^{\prime}$ einen binären nicht entarteten Teilraum von $\psi$ aufspannen, wenn eine der beiden folgenden stärkeren Voraussetzungen erfüllt ist:

c) $\operatorname{dim} \psi \geqq 3$, A hat weder $\mathbb{F}_{2}$ noch $\mathbb{F}_{3}$ als Restklassenkörper;

d) $\operatorname{dim} \psi=2$, A hat keinen der Körper $\mathbb{F}_{2}, \mathbb{F}_{3}, \mathbb{F}_{5}$ als Restklassenkörper.

Beweis. Wie zu Anfang des Beweises von Lemma 4.1 sehen wir, daß wir $A$ als Körper voraussetzen dürfen, und daß es genügt, einen isotropen Vektor

$$
z^{\prime}=x^{\prime} \otimes e+y^{\prime} \otimes f
$$

anzugeben, dessen Komponenten $x^{\prime}, y^{\prime}$ den im Lemma aufgezählten Bedingungen genügen. Wir wollen zunächst Teil (i) beweisen und setzen also im Falle $A=\mathbb{F}_{3}$ voraus, daß $\operatorname{dim} \psi \geqq 3$ ist. Aufgrund von Lemma 4.1 nehmen wir an, daß

$$
z=x \otimes e+y \otimes f
$$

ist mit anisotropen Vektoren $x, y$ aus $\psi$. Ist $b=0$, so ist $z^{\prime}:=y \otimes f$ ein isotroper Vektor, der allen in Teil (i) des Lemmas gestellten Forderungen genügt.

$\mathrm{Ab}$ jetzt sei $b \neq 0$. Angenommen, es ist

$$
2(x, y)+n(y)=0
$$

und insbesondere Char $A \neq 2$. Indem wir die Bilinearform von $\psi$ um einen skalaren Faktor abändern, können wir

$$
(x, y)=1, \quad n(y)=-2
$$

voraussetzen. Wir wollen nun $z$ an dem Vektor $y \otimes f$ spiegeln. Es ist

$$
q(y \otimes f)=-2 b, \quad B(z, y \otimes f)=1-4 b \neq 0 .
$$

Anwendung von $\tau(y \otimes f)$ auf $z$ ergibt also

$$
z^{\prime}=x \otimes e+\lambda y \otimes f
$$

mit $\lambda=(1-2 b)(2 b)^{-1}$. Sicherlich ist $\lambda \neq 0$, denn anderenfalls wäre

$$
n(x)=q\left(z^{\prime}\right)=q(z)=0 .
$$

im Widerspruch zu unserer Annahme. Ersichtlich ist auch $\lambda \neq 1$, weil $4 b \neq 1$ ist. Somit ist

$$
2(x, \lambda y)+n(\lambda y)=2\left(\lambda-\lambda^{2}\right) \neq 0 .
$$

Damit ist Teil i) des Lemmas bewiesen.

Wir kommen jetzt zum Beweis von Teil (ii) und dürfen schon voraussetzen, $\mathrm{da} B$

$$
z=x \otimes e+y \otimes f
$$

ist mit $n(y)=1,2(x, y)+n(y) \neq 0$. Die Körper $\mathbb{F}_{2}$ und $\mathbb{F}_{3}$ schließen wir aus. Wir haben eine Zerlegung

$$
x=\alpha y+u
$$


mit zu $y$ senkrechtem $u$ und $2 \alpha+1 \neq 0$. Ist $u$ anisotrop, so sind wir fertig, denn dann spannen $x$ und $y$ einen nicht ausgearteten Raum auf. Sei jetzt $n(u)=0$. Dann ist auch der Vektor

$$
\tilde{z}:=\alpha y \otimes e+y \otimes f
$$

isotrop. Wir betrachten zunächst den Fall, daß es in $\psi$ einen zu $y$ senkrechten anisotropen Vektor $w$ gibt. Das ist bei Char $A \neq 2$ automatisch der Fall. Wir spiegeln $\tilde{z}$ an dem Vektor

$$
g=(\lambda w+y) \otimes e
$$

mit geeignet zu wählendem $\lambda$. Das ist aussichtsreich, weil von vornherein

$$
B(g, \tilde{z})=2 \alpha+1 \neq 0
$$

ist. Zunächst haben wir

$$
q(g)=\lambda^{2} n(w)+1 \neq 0
$$

zu fordern. Wir erhalten

mit

$$
\tau(g)(\tilde{z})=(\gamma y+\delta w) \otimes e+y \otimes f=x^{\prime} \otimes e+y \otimes f
$$

$$
\begin{aligned}
& \gamma=q(g)^{-1}\left[\alpha \lambda^{2} n(w)-\alpha-1\right], \\
& \delta=-q(g)^{-1} \lambda(2 \alpha+1) .
\end{aligned}
$$

Ist nun $\lambda \neq 0$, so spannen $x^{\prime}$ und $y$ den nicht entarteten Raum $A y+A w$ auf. Schließlich besagt die Forderung $2\left(x^{\prime}, y\right)+n(y) \neq 0$, daß

$$
\lambda^{2} n(w)-1 \neq 0
$$

ist, wie man nachrechnet. Die drei an $\lambda$ gestellten Forderungen lassen sich erfüllen, falls $A$ mehr als 5 Elemente enthält und auch für $A=\mathbb{F}_{4}$. Im Falle $A=\mathbb{F}_{5}$ müssen wir $\operatorname{dim} \psi \geqq 3$ voraussetzen. Dann ist der Teilraum $(A y)^{\perp}$ von $\psi$ universell. Wir wählen jetzt obiges $w$ so, daß $n(w)=2$ ist. Dann werden die an $\lambda$ gestellten Forderungen durch $\lambda=1$ erfüllt.

Abschließend betrachten wir den Fall, daß in $\psi$ kein zu $y$ senkrechter anisotroper Vektor existiert. Jetzt hat $A$ die Charakteristik 2. Sei $\left\{g_{1}, \ldots, g_{r}\right\}$ eine Orthogonalbasis von $\psi$ und

$$
y=\lambda_{1} g_{1}+\cdots+\lambda_{r} g_{r} .
$$

Weil es keinen zu $y$ senkrechten anisotropen Vektor gibt, müssen alle $\lambda_{i} \neq 0$ sein. Indem wir die $g_{i}$ zu $\lambda_{i} g_{i}$ abändern, dürfen wir

$$
y=g_{1}+\cdots+g_{r}
$$

annehmen. Sei $a_{i}:=n\left(g_{i}\right)$. Für beliebige Indizes $i<j$ steht der Vektor $a_{i} g_{j}-a_{j} g_{i}$ auf $y$ senkrecht und ist somit isotrop. Daraus folgt $a_{i}=a_{j}$. Es ist also

$$
1=n(y)=r a_{1} .
$$

Somit hat $\psi$ ungerade Dimension und alle $a_{i}$ haben den Wert 1. Der Vektor

$$
z^{\prime \prime}:=\alpha g_{1} \otimes e+g_{1} \otimes f
$$

hat nun wieder die Länge Null, und es gibt in $\psi$ zu $g_{1}$ senkrechte anisotrope Vektoren. Unser ursprünglicher Vektor $z$ läßt sich durch eine eigentliche Iso- 
metrie in $z^{\prime \prime}$ transformieren und dann in einen Vektor $x^{\prime} \otimes e+y^{\prime} \otimes f$ mit den gewünschten Eigenschaften, wie weiter oben gezeigt wurde.

Jetzt ist Lemma 5.3 und damit Satz 5.2 völlig bewiesen. Man prüft übrigens leicht, daß die dort aufgezählten Einschränkungen für die Restklassenkörper von $\boldsymbol{A}$ wirklich erforderlich sind.

Schon aus Satz 5.1 und unserer Beschreibung des Annullators $\mathfrak{A}(\varphi)$ von $\varphi$ in dem $W(A)$-Modul der quadratischen Räume gerader Dimension in Satz 3.4 läßt sich eine interessante Folgerung ziehen.

Satz 5.4. Sei $\rho$ ein weiterer bilinearer Pfisterraum und sei

$$
\varphi \cong(a) \otimes \rho \perp \psi
$$

mit $a$ in $A^{*}$ und einem Raum $\psi$. Dann ist der Annullator $\mathfrak{A}(\rho)$ in $\mathfrak{A}(\varphi)$ enthalten.

Beweis. Weil a Ähnlichkeitsnorm von $\varphi$ ist, dürfen wir in der Zerlegung (*) $a=1$ annehmen. $\rho$ ist also Teilraum von $\varphi$ und somit $\rho^{\prime}$ Teilraum von $\varphi^{\prime}$. Die Behauptung folgt jetzt aus der Beschreibung von $\mathfrak{X}(\varphi)$ und $\mathfrak{A}(\rho)$ durch die Sätze 3.4 und 5.1. q.e.d.

Aufgrund dieses Satzes ist die Vermutung naheliegend, daß in der Situation (*) ein Raum $\sigma$ existiert mit $\varphi \cong \rho \otimes \sigma$ oder wenigstens $\varphi \sim \rho \otimes \sigma$. Kürzlich hat $H$. Bauer (Regensburg) festgestellt, daß im Falle $2 \in A^{*}$ in der Tat eine Faktorisierung $\varphi \cong \rho \otimes \sigma$ existiert. Man kann dann sogar $\dot{\sigma}$ als Pfisterraum wählen. Im Körperfall findet sich dieses Resultat schon bei Elman und Lam [5].

\section{§6. Der Hauptsatz}

Wir beweisen jetzt eine Verschärfung des früheren Satzes 3.4.

Hauptsatz 6.1. $A$ habe nicht $\mathbb{F}_{2}$ als Restklassenkörper. $\varphi$ sei ein bilinearer Pfisterraum einer Dimension $>1$. Dann wird der Annullator $\mathfrak{A}(\varphi)$ von binären Räumen erzeugt. (Für eine Beschreibung dieser Räume s. § 5.)

Für $\operatorname{dim}(\varphi)=2$ wurde dies schon in Kor. 3.3 festgestellt. Im folgenden nehmen wir stets $\operatorname{dim}(\varphi) \geqq 4$ an. Sei $\mathfrak{B}(\varphi)$ der von den Räumen vom Typ 1 erzeugte $W(A)$ Teilmodul von $\mathfrak{U}(\varphi)$. Aufgrund von Satz 3.4 genügt es einzusehen, daß jeder Raum $F$ vom Typ 2 in $\mathfrak{B}(\varphi)$ liegt. Sei also

$$
F=(1,-\alpha) \otimes E,
$$

wobei $E$ ein binärer Raum $[1, b]$ ist und $\alpha$ von $\varphi \otimes E$ dargestellt wird. Aufgrund von Lemma 4.3 gibt es Vektoren $x$ und $y$ in $\varphi$ mit $n(y)$ Einheit und

$$
\alpha=n(x)+(x, y)+n(y) b .
$$

\{Nach Lemma 4.1 kann man überdies $n(x) \in A^{*}$ erreichen, doch brauchen wir das nicht.\} Mit $z:=n(y)^{-1} y$ gilt $\alpha=n(y) \beta$, wobei

$$
\beta=b+(x, z)+n(x) n(z)
$$

ist. Wir können nun einen Vektor $u$ in $\varphi^{\prime}$ finden, so da $\beta$

$$
n(x) n(z)=(x, z)^{2}+n(u)
$$


ist [2, vgl. Beweis von Satz 5.1]. Mit der Abkürzung

haben wir also

$$
\gamma:=(x, z)=n(y)^{-1}(x, y)
$$

Nun gilt

$$
\beta=b+\gamma+\gamma^{2}+n(u)
$$

$$
(1,-\alpha) \sim(1,-n(y)) \perp(n(y)) \otimes(1,-\beta) .
$$

Es genügt also zu zeigen, daß die Räume

und

$$
F_{1}=(1,-n(y)) \otimes[1, b]
$$

$$
F_{2}=(1,-\beta) \otimes[1, b]
$$

in $\mathfrak{B}(\varphi)$ liegen. Man zieht sich überdies leicht auf den Fall $n(y)=\lambda^{2}+n(v)$ mit strikt anisotropen $v \in \varphi^{\prime}$ zurück $^{1} . F_{1}$ wird von $(1, n(v))$ annulliert. Aufgrund von Satz 3.1 und Satz 5.1 ist also $F_{1}$ orthogonale Summe von zwei Räumen, die von $(1, n(v))$ annulliert werden. Nach Satz 5.4 werden diese Räume auch von $\varphi$ annulliert, und liegen somit in $\mathfrak{B}(\varphi)$. Wir untersuchen jetzt den Raum $F_{2}$. Angenommen, es ist $n(u)$ eine Einheit. Dann kommen wir wie folgt zum Ziel: $F_{2}$ stellt das Element

$$
\gamma^{2}+\gamma+b-\beta=-n(u)
$$

dar, also ist $F_{2} \cong(-n(u)) \otimes F_{2}$, und $F_{2}$ wird von $(1, n(u))$ annulliert. Daraus folgt wie zuvor, daß $F_{2}$ in $\mathfrak{B}(\varphi)$ liegt. Nun ist

$$
n(u)=n(y)^{-2}\left[n(x) n(y)-(x, y)^{2}\right] .
$$

Der Beweis des Hauptsatzes ist also erbracht, wenn wir das folgende Lemma gezeigt haben.

Lemma 6.2. $A$ habe nicht $\mathbb{F}_{2}$ als Restklassenkörper. Wir betrachten wieder die vor Lemma 4.1 beschriebene Situation. Jetzt sei $\operatorname{dim} \psi \geqq 3, q(e)=1$ und $z$ strikt anisotrop. Dann gibt es einen eigentlichen Automorphismus $\sigma$ von $\psi \otimes E$, so daß $\sigma(z)$ die Gestalt $x^{\prime} \otimes e+y^{\prime} \otimes f$ hat mit $n\left(y^{\prime}\right)$ Einheit und $n\left(x^{\prime}\right) n\left(y^{\prime}\right)-\left(x^{\prime}, y^{\prime}\right)^{2}$ Einheit.

Beweis. Wir dürfen voraussetzen, daß $A$ Körper ist, und brauchen nur irgendeinen Vektor $z^{\prime}=x^{\prime} \otimes e+y^{\prime} \otimes f$ der soeben beschriebenen Gestalt von gleicher Länge wie $z$ anzugeben. Den Wert $q(f)$ bezeichnen wir wieder mit $b$.

Wir diskutieren zunächst den Fall $b=0$. Ist $A$ endlich, so wählen wir für $y^{\prime}$ irgendeinen Vektor aus einer Orthogonalbasis von $\psi$. Dann wählen wir für $x^{\prime}$ einen zu $y^{\prime}$ senkrechten Vektor mit $n\left(x^{\prime}\right)=q(z)$. Das ist möglich, weil der Raum $\left(A y^{\prime}\right)^{\perp}$ universell ist. Ist $A$ unendlich, so wählen wir zwei zueinander senkrechte anisotrope Vektoren $u, v$ und kommen mit dem Ansatz

$$
x^{\prime}=u, \quad y^{\prime}=\lambda u+\mu v
$$

für geeignete $\lambda$ und $\mu$ aus $A, \mu \neq 0$, zum Ziel, vgl. Anfang des Beweises von Lemma 4.1.

Weiter erledigen wir vorweg den Fall, daß $A$ endlich ist. Wir wählen in $\psi$ einen anisotropen Vektor $u$, der sich zu einer Orthogonalbasis von $\psi$ ergänzen

1 Mit Hilfe von [2] findet man einen strikt anisotropen Vektor $w$ in $\varphi^{\prime}$, so daß $n(y) n(w)=\lambda^{2}+n(v)$ mit strikt anisotropem $v$ in $\varphi^{\prime}$. Man benutze nun eine zu (*) analoge Relation. 
läßt, und machen den Ansatz

$$
x^{\prime}=\lambda u+v, \quad y^{\prime}=u
$$

mit $v$ anisotrop und senkrecht zu $u$. Wir haben dann nur die weitere Bedingung

$$
\left(\lambda^{2}+\lambda+b\right) n(u)+n(v)=q(z)
$$

zu erfüllen. Das ist für $A \neq \mathbb{F}_{2}$ möglich:

Man wähle zunächst ein $\lambda$ in $A$ mit

$$
\lambda^{2}+\lambda+b \neq n(u)^{-1} q(z)
$$

und dann in dem universellen Teilraum $(A u)^{\perp}$ von $\psi$ einen Vektor $v$, so daß (*) gilt.

Sei ab jetzt $b \neq 0$ und $A$ unendlich. Dann gehen wir genauso wie im Beweis von Teil (ii) des Lemmas 5.3 vor. Aufgrund von Lemma 4.1 dürfen wir

$$
z=x \otimes e+y \otimes f
$$

mit anisotropen $x, y$ voraussetzen und dann sogar $n(y)=1$ annehmen. Wir haben eine Zerlegung $x=\alpha y+u$ mit $\alpha \neq 0$ und zu $y$ senkrechtem $u$. Ist $u$ anisotrop, so sind wir fertig. Sei jetzt $u$ isotrop. Dann hat $z$ dieselbe Länge wie der Vektor

$$
\tilde{z}:=\alpha y \otimes e+y \otimes f .
$$

Aufgrund derselben Überlegung wie am Ende des Beweises von Lemma 5.3 können wir uns auf den Fall zurückziehen, daß es in $\psi$ einen zu $y$ senkrechten anisotropen Vektor $w$ gibt. Ist $2 \alpha+1 \neq 0$, so spiegeln wir $\tilde{z}$ an einem Vektor

$$
g=(\lambda w+y) \otimes e,
$$

und dieselbe Rechnung wie im Beweis von Lemma 5.3 (ii) zeigt, daß bei geeigneter Wahl von $\lambda$ der Vektor $g$ anisotrop ist und $z^{\prime}=\tau(g)(\tilde{z})$ die gewünschte Gestalt hat. Ist $2 \alpha+1=0$, so spiegeln wir $\tilde{z}$ an einem Vektor

$$
h:=(\lambda w+y) \otimes f
$$

mit geeignetem $\lambda$. Wir haben $\lambda^{2} n(w)+1 \neq 0$ zu fordern, damit $h$ anisotrop ist. Es ist

$$
B(h, \tilde{z})=B\left(-\frac{1}{2} e+f, f\right)=\frac{1}{2}(4 b-1) \neq 0 .
$$

Ist nun $\lambda \neq 0$, so erhält man

$$
\tau(h)(\tilde{z})=-\frac{1}{2} y \otimes e+(\gamma y+\delta w) \otimes f
$$

mit $\delta \neq 0$. Die Forderung, da $\beta \gamma y+\delta w$ anisotrop ist, bedeutet weiter, da $\beta \lambda$ nicht Wurzel eines gewissen Polynoms vom Grad 4 ist. Weil $A$ unendlich ist, können wir alle hier an $\lambda$ gestellten Wünsche erfüllen. Damit ist unser Lemma 6.2 und Satz 6.1 bewiesen.

Man sieht übrigens leicht ein, daß unsere Voraussetzung über $A$ in Lemma 6.2 notwendig ist. 


\section{Literatur}

1. Baeza, R.: Über die Torsion der Wittgruppe $W q(A)$ eines semi-lokalen Ringes. Math. Ann. 207, $121-131$ (1974)

2. Baeza, R.: Eine Bemerkung über Pfisterformen, erscheint in Arch. Math.

3. Bass, H.: Lectures on topics in algebraic $K$-theory. Bombay: Tata Institute of Fundamental Research 1967

4. Elman, R., Lam, T.Y.: Pfister forms and $K$-theory of fields. J. Algebra 23, $181-213$ (1972)

5. Elman, R., Lam, T.Y.: Quadratic forms and the $u$-invariant I. Math. Z. 131, 283-304 (1973)

6. Knebusch, M.: Runde Formen über semilokalen Ringen. Math. Ann. 193, 21 - 34 (1971)

7. Knebusch, M.: Generalization of a theorem of Artin-Pfister to arbitrary semi-local rings, and related topics; erscheint in J. Algebra

8. Knebusch, M.: Isometrien über semilokalen Ringen. Math. Z. 108, 255-268 (1969)

9. Knebusch, M.: Bemerkungen zur Theorie der quadratischen Formen über semilokalen Ringen. Schriften Math. Inst. Universität des Saarlandes, Saarbrücken 1969 (auf Wunsch beim Autor erhältlich)

10. Witt, E.: Theorie der quadratischen Formen in beliebigen Körpern. J. reine angew. Math. 176, $31-44$ (1937)

Ricardo Baeza

Mathematisches Institut der

Universität des Saarlandes

D-6600 Saarbrücken

Bundesrepublik Deutschland

\author{
Manfred Knebusch \\ Mathematisches Institut der \\ Universität Regensburg \\ D-8400 Regensburg \\ Bundesrepublik Deutschland
}

